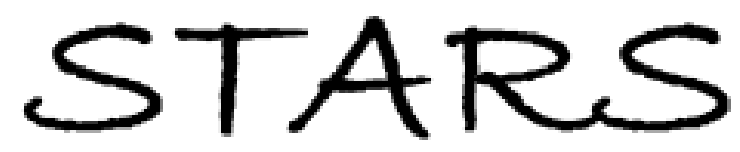

University of Central Florida

STARS

$1-1-2008$

\title{
Dynamics of DNA translocation through an attractive nanopore
}

Kaifu Luo

Tapio Ala-Nissila

See-Chan Ying

Aniket Bhattacharya

Find similar works at: https://stars.library.ucf.edu/facultybib2000

University of Central Florida Libraries http://library.ucf.edu

This Article is brought to you for free and open access by the Faculty Bibliography at STARS. It has been accepted for inclusion in Faculty Bibliography 2000s by an authorized administrator of STARS. For more information, please contactSTARS@ucf.edu.

\section{Recommended Citation}

Luo, Kaifu; Ala-Nissila, Tapio; Ying, See-Chan; and Bhattacharya, Aniket, "Dynamics of DNA translocation through an attractive nanopore" (2008). Faculty Bibliography 2000s. 659.

https://stars.library.ucf.edu/facultybib2000/659

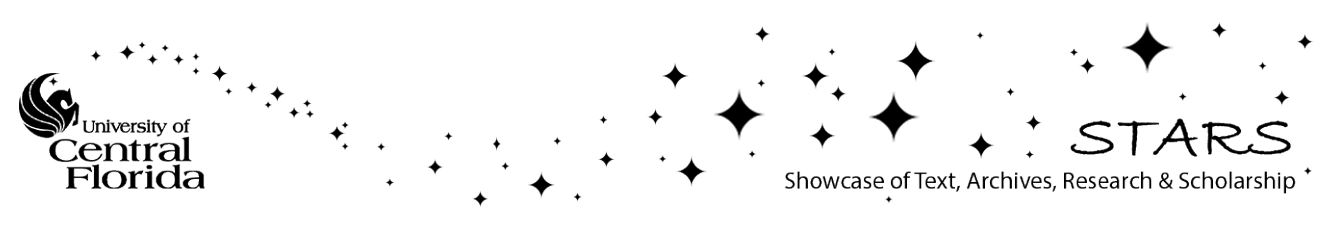




\title{
Dynamics of DNA translocation through an attractive nanopore
}

\author{
Kaifu Luo, ${ }^{1,2, *}$ Tapio Ala-Nissila, ${ }^{1,3}$ See-Chen Ying, ${ }^{3}$ and Aniket Bhattacharya ${ }^{4}$ \\ ${ }^{1}$ Department of Applied Physics, Helsinki University of Technology, P.O. Box 1100, FIN-02015 TKK, Espoo, Finland \\ ${ }^{2}$ Physics Department, Technical University of Munich, D-85748 Garching, Germany \\ ${ }^{3}$ Department of Physics, Box 1843, Brown University, Providence, Rhode Island 02912-1843, USA \\ ${ }^{4}$ Department of Physics, University of Central Florida, Orlando, Florida 32816-2385, USA
}

(Received 20 August 2008; published 9 December 2008)

\begin{abstract}
We investigate the dynamics of single-stranded DNA translocation through a nanopore driven by an external force using Langevin dynamics simulations in two dimensions to study how the translocation dynamics depend on the details of the DNA sequences. We consider a coarse-grained model of DNA built from two bases $A$ and $C$, having different base-pore interactions, e.g., a strong (weak) attractive force between the pore and the base $A(C)$ inside the pore. From a series of studies on hetero-DNAs with repeat units $A_{m} C_{n}$, we find that the translocation time decreases exponentially as a function of the volume fraction $f_{C}$ of the base $C$. For longer $A$ sequences with $f_{C} \leqslant 0.5$, the translocation time strongly depends on the orientation of DNA, namely which base enters the pore first. Our studies clearly demonstrate that for a DNA of certain length $N$ with repeat units $A_{m} C_{n}$, the pattern exhibited by the waiting times of the individual bases and their periodicity can unambiguously determine the values of $m, n$, and $N$, respectively. Therefore, a prospective experimental realization of this phenomenon may lead to fast and efficient sequence detection.
\end{abstract}

DOI: 10.1103/PhysRevE.78.061911

PACS number(s): 87.15.A-, 87.15.H-

\section{INTRODUCTION}

Polymer translocation through a nanopore is a challenging problem in polymer physics and it also plays a critical role in numerous biological processes, such as DNA and RNA translocation across nuclear pores, protein transport through membrane channels, and virus injection.

In a seminal experimental paper, Kasianowicz et al. [1] demonstrated that an electric field can drive single-stranded DNA and RNA molecules through the water-filled $\alpha$-hemolysin channel and that the passage of each molecule is signaled by a blockade in the channel current. These observations can be directly used to characterize the polymer length. Triggered by their experiments and various potential technological applications $[1,2]$, such as rapid DNA sequencing, gene therapy, and controlled drug delivery, the polymer translocation has become a subject of intensive experimental [3-22] and theoretical [21-71] studies.

One of the fundamental questions that has been addressed in the community is how the translocation time $\tau$ depends on the system parameters and the polymer characteristics, such as chain length $N$ $[5,6,21,22,24,27,39,47,48,50-58,60,67]$, sequence and secondary structure $[3,4,6,9,38,40,57-59,70,71]$, pore length $L$ and width $W$ [53], driving force $F$ $[5,6,9,11,48,48,54-58,61,62,67]$, and polymer-pore interactions $[4,6,15,39,58,59,67]$. A central issue from the point of view of sequencing is whether DNA translocation through a nanopore can be used to determine the detailed sequence structure of the molecule [59].

It has been demonstrated experimentally $[4,6,15]$ that DNA translocation dynamics is strongly influenced by

\footnotetext{
*Author to whom correspondence should be addressed; luokaifu@gmail.com
}

nucleotide-pore interactions. Intuitively, for sufficiently strong attraction, the residence time of each monomer in the pore should increase, resulting in a much longer translocation time. In a recent paper, we have investigated the influence of base-pore interaction on the translocation dynamics using Langevin dynamics simulations [58]. The results show that an attractive interaction increases translocation time slowly for weak attraction while an exponential increase is observed for strong attraction in the activated regime [67]. Under weak driving force and strong attractive force, the translocation time shows nonmonotonic behavior. Our results are in good agreement with experimental findings $[4,6,15]$.

While a DNA is composed of four different nucleotides [72], to date most of the theoretical treatments have focused on scaling and universal aspects of translocation of a homogeneous polymers, although several experiments [2,9] show that in the real biological systems inhomogeneities in the structure and interactions between polymer and other molecules might have a significant effect on the overall dynamics. Previously, we have considered heteropolymers consisting of two types of monomers labeled $A$ and $C$, which are distinguished by the magnitude of the driving force that they experience inside the pore [57]. This model captured some essential features of the heteropolymer translocation. However, for real biopolymers such as DNA and RNA, no charge difference between the monomers (nucleotides) exists. Instead, the nucleotide-pore interactions are base specific $[4,6]$. Thus, a more realistic model for studying the influence of structure on biopolymers translocation should differentiate bases $A$ and $C$ by different base-pore interactions. In a recent paper [59], we have adopted such a model and investigated hetero-DNAs with symmetric blocks using Langevin dynamics simulations. We found that the translocation time depends strongly on the block length as well as on the orientation of which base enters the pore first.

In this paper we extend our results for the single-stranded DNA composed of symmetric blocks, furnish more general 


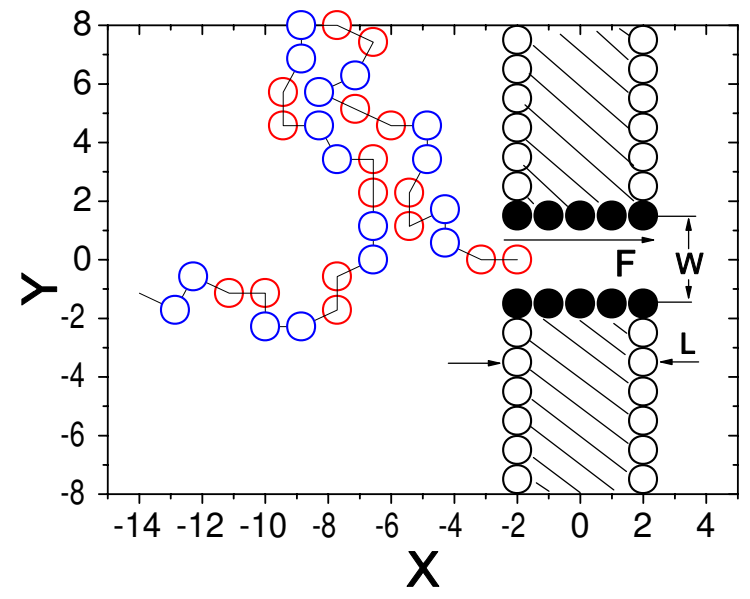

FIG. 1. (Color online) A schematic representation of the system. The pore length $L=5$ and the pore width $W=3$.

characteristics of the residence time inside the pore, and provide more comprehensive results for the translocation dynamics of various sequences of a two component heteroDNA. We also provide a simple interpretation for the sequence dependence of the monomer waiting time distribution and the total translocation time. The paper is organized as follows. In Sec. II, we briefly describe our model and the simulation technique. In Sec. III, we present our results. Finally, the conclusions and discussion are presented in Sec. IV.

\section{MODEL AND METHOD}

In our numerical simulations, the polymer chains are modeled as bead-spring chains. Excluded volume interaction between monomers is modeled by a short-range repulsive Lennard-Jones (LJ) potential: $U_{\mathrm{LJ}}(r)=4 \varepsilon\left[\left(\frac{\sigma}{r}\right)^{12}-\left(\frac{\sigma}{r}\right)^{6}\right]+\varepsilon$ for $r \leqslant 2^{1 / 6} \sigma$ and 0 for $r>2^{1 / 6} \sigma$. Here, $\sigma$ is the diameter of a monomer, and $\varepsilon$ is the depth of the potential. The connectivity between neighboring monomers is modeled as a finite extension nonlinear elastic (FENE) spring with $U_{\mathrm{FENE}}(r)$ $=-\frac{1}{2} k R_{0}^{2} \ln \left(1-r^{2} / R_{0}^{2}\right)$, where $r$ is the distance between consecutive monomers, $k$ is the spring constant and $R_{0}$ is the maximum allowed separation between connected monomers.

We consider a two-dimensional (2D) geometry as shown in Fig. 1, where the walls along the $y$ direction are formed by stationary particles within a distance $\sigma$ from each other. The pore of length $L$ and width $W$ are formed from two rows of stationary particles represented by black circles in Fig. 1. Between all monomer-wall particle pairs, there exists the same short-range repulsive LJ interaction as described above. The base-pore interaction is modeled by a LJ potential with a cutoff of $2.5 \sigma$ and interaction strength $\varepsilon_{p A}$ for the base $A$ and $\varepsilon_{p C}$ for the base $C$. This interaction can be either attractive or repulsive depending on the position of the monomer from the pore particles.

In the Langevin dynamics simulation, each monomer is subjected to conservative, frictional, and random forces, respectively, with [73] $m \ddot{\mathbf{r}}_{i}=-\nabla\left(U_{\mathrm{LJ}}+U_{\mathrm{FENE}}\right)+\mathbf{F}_{\mathrm{ext}}-\xi \mathbf{v}_{i}+\mathbf{F}_{i}^{R}$, where $m$ is the monomer's mass, $\xi$ is the friction coefficient, $\mathbf{v}_{i}$ is the monomer's velocity, and $\mathbf{F}_{i}^{R}$ is the random force which satisfies the fluctuation-dissipation theorem. The external force is expressed as $\mathbf{F}_{\text {ext }}=F \hat{x}$, where $F$ is the external force strength exerted on the monomers in the pore, and $\hat{x}$ is a unit vector in the direction along the pore axis.

The LJ parameters $\varepsilon, \sigma$, and the bead mass $m$ fix the system energy, length, and mass units respectively, leading to the corresponding time scale $t_{\mathrm{LJ}}=\left(m \sigma^{2} / \varepsilon\right)^{1 / 2}$ and force scale $\varepsilon / \sigma$. In our model, each bead corresponds to a Kuhn length of a single-stranded DNA containing approximately three nucleotide bases, so the value of $\sigma \approx 1.5 \mathrm{~nm}$ [74]. The average mass of a base in DNA is about $312 \mathrm{amu}$, so the bead mass $m \approx 936$ amu. We set $k_{B} T=1.2 \varepsilon$, so the interaction strength $\varepsilon$ corresponds to $3.39 \times 10^{-21} \mathrm{~J}$ at a temperature $295 \mathrm{~K}$. This leads to a time scale of $32.1 \mathrm{ps}$ and a force scale of $2.3 \mathrm{pN}$. The remaining dimensionless parameters in the model are chosen to be $R_{0}=2, k=7, \xi=0.7$. In addition, the driving force for a bead in the pore is set as $F=0.5$ unless otherwise stated. This value corresponds to a voltage of about $187.9 \mathrm{mV}$ across the pore (assuming three unit charges on a bead and the effective charge $0.094 e$ for a unit charge $[9,10])$, within the range of experimental parameters $[1,2,4-6]$. The pore width $W=3$. This ensures that monomers $A$ and $C$ encounter an attractive force inside the pore [75]. The base-pore interactions $\varepsilon_{p A}=3.0$ and $\varepsilon_{p C}=1.0$ are chosen based on comparison of the theoretical results [58] with the experimental data [4] for the translocation time distribution histogram of poly $(\mathrm{dC})_{100}$ and poly $(\mathrm{dA})_{100}$. It is worthwhile to note that the translocation time depends strongly on the pore length $L$. We have checked that $L \approx 10 \mathrm{~nm}$ produces average translocation times $\tau \approx 100 \mu \mathrm{s}$ in accordance with the experimental data [4]. For computational efficiency we present results for $L=5(7.5 \mathrm{~nm})$ here. The Langevin equation is integrated in time by a method described by Ermak and Buckholz [76] in 2D.

To create the initial configuration, the first monomer of the chain is placed in the entrance of the pore. The polymer is then allowed to relax to obtain an equilibrium configuration. The translocation time is defined as the time interval between the entrance of the first bead into the pore and the exit of the last bead. The estimate for the translocation time was obtained by neglecting any failed translocation and then calculating the average duration of the successful translocation. Typically, we average our data over 2000 independent runs with different initial conditions.

The present model has some limitations, which should be discussed here. Due to the coarse-grained nature of the model, it is clear that the quantitative details of some results presented here depend on the microscopics of the real-world experimental setup. However, we have done some additional checks to ensure that our main conclusions remain unaffected. For example, we have carried out some simulations in three dimensions (3D) and checked that the dimensionality plays an unimportant role here. Regarding the issue of hydrodynamics, which is also neglected in our model, recent molecular dynamics [33] and lattice Boltzmann [34,35] simulation results show that hydrodynamics is screened out in a narrow pore, which is the case here and in the experiments. Finally, for the present case where we model singlestranded DNA chains, the bending stiffness of the chain is also not expected to play a role. 


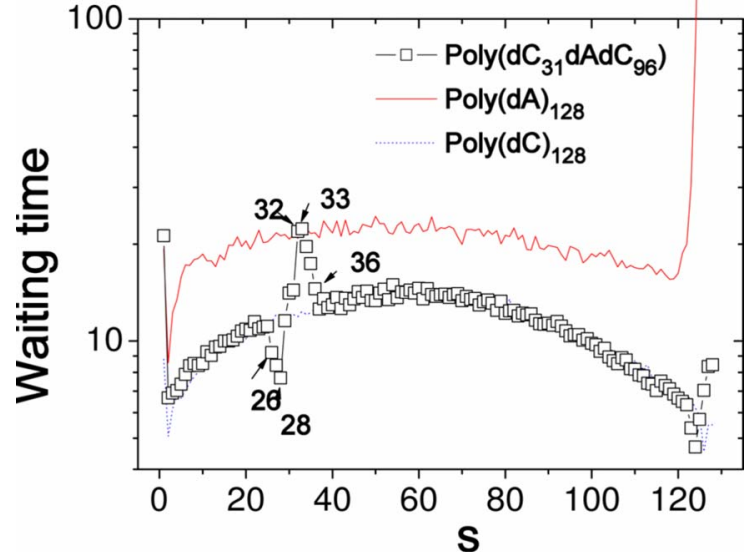

FIG. 2. (Color online) The waiting time distribution for poly $\left(\mathrm{dC}_{31} \mathrm{dAdC}_{96}\right)$. Here $\varepsilon_{p A}=3.0, \varepsilon_{p C}=1.0$.

\section{RESULTS AND DISCUSSIONS}

\section{A. Recognizing position-specific base $A$ substitutions in $\operatorname{poly}(\mathrm{dC})$}

As demonstrated by experiments [1,2], a nanopore might be used for DNA sequencing. The most fundamental concern has been whether $\alpha$-hemolysin or any other natural or synthetic nanopore is capable of recognizing DNA with single base resolution.

To address this question, we first investigate the translocation of poly $(\mathrm{dC})$ with a single position-specific base $A$ substitution. Without any loss of generality, we present here only results for chain length $N=128$, as many of the main conclusions here are not dependent on the chain length. Figure 2 shows the waiting time distribution for poly $\left(\mathrm{dC}_{31} \mathrm{dAdC}_{96}\right)$. The waiting time of the monomer $s$ is defined as the average time between the events that monomer $s$ and monomer $s+1$ exit the pore $[54,55,57]$. It yields more detailed information for the translocation process than the overall translocation time. Compared with the waiting time distribution for poly $\left(\mathrm{dC}_{128}\right)$, the waiting times for $s=26-36$ are different, where one minimum $(s=28)$ and maximum $(s=32)$ are observed. This result can be easily understood through the following consideration. The pore of length $L=5$ can accommodate $n_{\text {pore }}=6$ monomers on the average. When the monomer $s=32-n_{\text {pore }}=26$ is at the end of the pore ready to exit to the trans side, the monomer corresponding to base $A$ has just entered the pore through the cis side. The presence of the $A$ monomer at this end of the pore reduces the probability of the backward motion of the polymer and hence the waiting time starts to decrease starting from $s=26$ until it reaches a minimum at $s=28$ when the $A$ monomer is about one-half way inside the pore, after which the waiting time of subsequent monomers leaving the pore starts to increase until it reaches $s=32$ which corresponds to the point where the monomer $A$ is at the end of the pore ready to exit to the cis side. This maximum in waiting time results from the activated nature of the escape of the monomer $A$ from the pore. After the monomer corresponding to base $A$ has translocated, the next few monomers still have longer waiting times compared with those in a homopolymer poly $\left(\mathrm{dC}_{128}\right)$. This is due

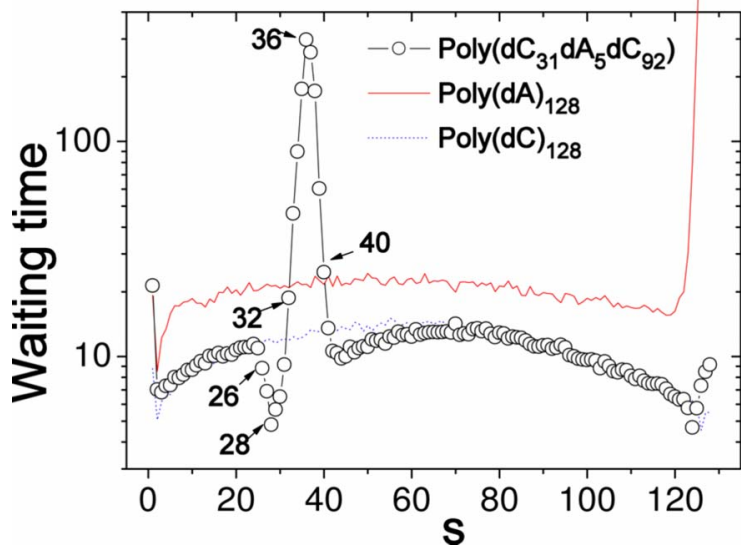

FIG. 3. (Color online) The waiting time distribution for poly $\left(\mathrm{dC}_{31} \mathrm{dA}_{5} \mathrm{dC}_{92}\right)$. Here $\varepsilon_{p A}=3.0, \varepsilon_{p C}=1.0$.

to the fact that the attraction between the pore and the monomer corresponding to base $A$ induces more backward events. For other position-specific single base $A$ substitutions, we observed similar results.

For longer base $A$ sequence substitutions such as poly $\left(\mathrm{dC}_{31} \mathrm{dA}_{5} \mathrm{dC}_{92}\right)$, the results are shown in Fig. 3. They can be understood following the same reasoning presented above for the single $A$ sequence. We still observe one minimum and one maximum between $s=26-36$. As expected, the beginning of the decrease in waiting time still starts at $s=26$ corresponding to $s=32-n_{\text {pore }}=26$. The maximum now changes to $s=31+5$ when the last monomer of type $A$ is at the end of the pore ready to exit to the trans side. For other sequences such as poly $\left(\mathrm{dC}_{31} \mathrm{dA}_{m} \mathrm{dC}_{97-m}\right)$, the start of the downturn at $s=26$ and the minimum at $s=28$ remains the same while the maximum will be shifted to $s=31+m$. Thus, we can conclude that if the waiting time distribution can be accurately determined, it allows identification of the sequence $A$ in a straightforward manner.

\section{B. Effect of the different composition on the translocation}

In this section, we present results for the translocation dynamics for hetero-DNAs with repeat units $A_{m} C_{n}$, where $m$ and $n$ are the length of the bases $A$ and $C$ in a single unit, respectively. We define $m+n$ as the block length of the polymer, and the volume fraction of the $C$ component as $f_{C}$ $=n /(m+n)$. In the translocation dynamics of these heteroDNAs, it turns out that we need to distinguish the two different cases where the base $A$ or the base $C$ first enters the pore, respectively.

We first examine short repeat units with $m+n \leqslant 8$ for the whole range of volume fractions $f_{C}$. The results are shown in Fig. 4. Because the attraction between the pore and the base $A$ is stronger, the total translocation time $\tau_{A}$ for poly $(\mathrm{dA})_{128}$ $\left(f_{C}=0\right)$ is longer than the corresponding value $\tau_{C}$ for a poly $(\mathrm{dC})_{128}$ as found in our previous work [58]. The solid curve in Fig. 4 represents a simple average of the translocation time for poly $(\mathrm{dA})_{128}$ and poly $(\mathrm{dC})_{128}$ given by $\tau\left(f_{C}\right)$ $=\tau_{A}\left(1-f_{C}\right)+\tau_{C} f_{C}$. It can be seen that the actual results for the translocation time of hetero-DNAs with short repeat units 


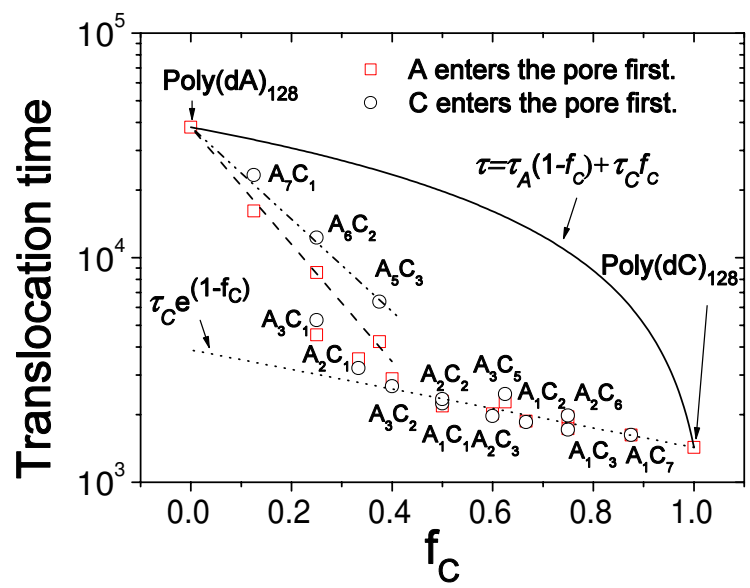

FIG. 4. (Color online) Translocation time for hetero-DNAs with repeat units $A_{m} C_{n}$ for different values of $m$ and $n$ as a function of the volume fraction of the base $C$. Here $\varepsilon_{p A}=3.0, \varepsilon_{p C}=1.0$, and the chain length $N=128$.

are always lower than this average, indicating a correlation effect between different monomer blocks. As expected, the translocation time decreases with increasing $f_{C}$. For $m \leqslant 3$ $\left(f_{C} \geqslant 0.6\right)$, the translocation time is almost independent on the orientation of which base enters the pore first. This is because the pore with length $L=5$ can accommodate more than three monomers. Thus, for $m \leqslant 3$, the translocation times for the hetero-DNAs are determined largely by the configurations where all the $A$ monomers are trapped inside the pore, independent of whether the base $A$ or $C$ enters the pore first. In this regime, the translocation time can be fitted empirically by the formula $\tau\left(f_{C}\right)=\tau_{C} e^{\left(1-f_{C}\right)}$.

For small values of $f_{C} \leqslant 0.4 \quad(m \geqslant 5)$, the translocation time $\tau$ does depend on the orientation, with $\tau$ being larger for the case where the base $C$ enters the pore first. This asymmetry is due to the fact that the translocation time is now controlled by the last block $A$ exiting the pore. When this is not followed by a block $C$, the activation barrier for the exit of the last block is large, leading to a much longer total translocation time. In this regime, $\tau$ shows faster exponential decay with increasing $f_{C}$ when the base $A$ enters the pore first. To quantify the asymmetry, we define $r$ as the ratio of translocation times for the case where base $C$ enters the pore first to the case where $A$ enters the pore first. Figure 5 shows $r$ for different sequences. It is clear that the asymmetry increase with the length of the base $A$ sequence.

Compared to the total translocation time, the waiting time for individual bases yields more information for the detailed sequence structure as shown in the last section. To show how the waiting time can be used to read a periodic sequence, we show the result for poly $\left(\mathrm{dA}_{5} \mathrm{dC}_{3}\right)_{16}$ in Fig. 6. There are several features worth noting. First, the waiting time of the last block $A$ is much longer than the rest, due to the activated nature of the last block $A$. This effect is more pronounced for $C$ entering the pore first, since it leads to a much larger activation energy for the exit of the last block. For the orientation in which $A$ enters the pore first, both the monomers in the last block $A$ and the last block $C$ exhibit longer waiting times, but not as strongly as the other orientation as the cor-

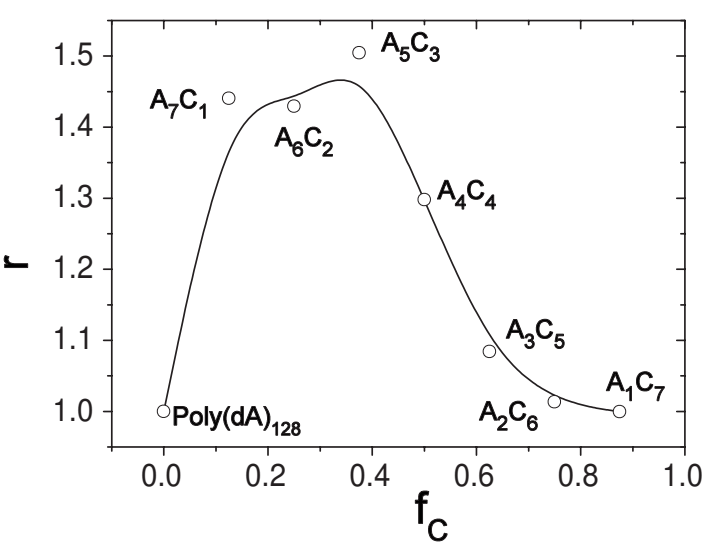

FIG. 5. $r$ as a function of the different composition for repeat units $A_{m} C_{n}$. Here, we define $r$ as the ratio of translocation times for base $C$ entering the pore first to base $A$ entering the pore first. Here, $\varepsilon_{p A}=3.0, \varepsilon_{p C}=1.0$, and the chain length $N=128$.

responding activation energies are smaller. The orientation of DNA can thus be differentiated in this case. Second, the sequence lengths $m=5$ for the base $A$ and $n=3$ for the base $C$ are clearly distinguished in comparison with the waiting time distributions of their homopolymers.

Moreover, we find that the waiting times for the ordered DNA with repeat units $A_{m} C_{n}$ exhibit "fringes" reminiscent of optical interference pattern. This is due to the fact that near the end of exit of any block $A$, the waiting time increases to

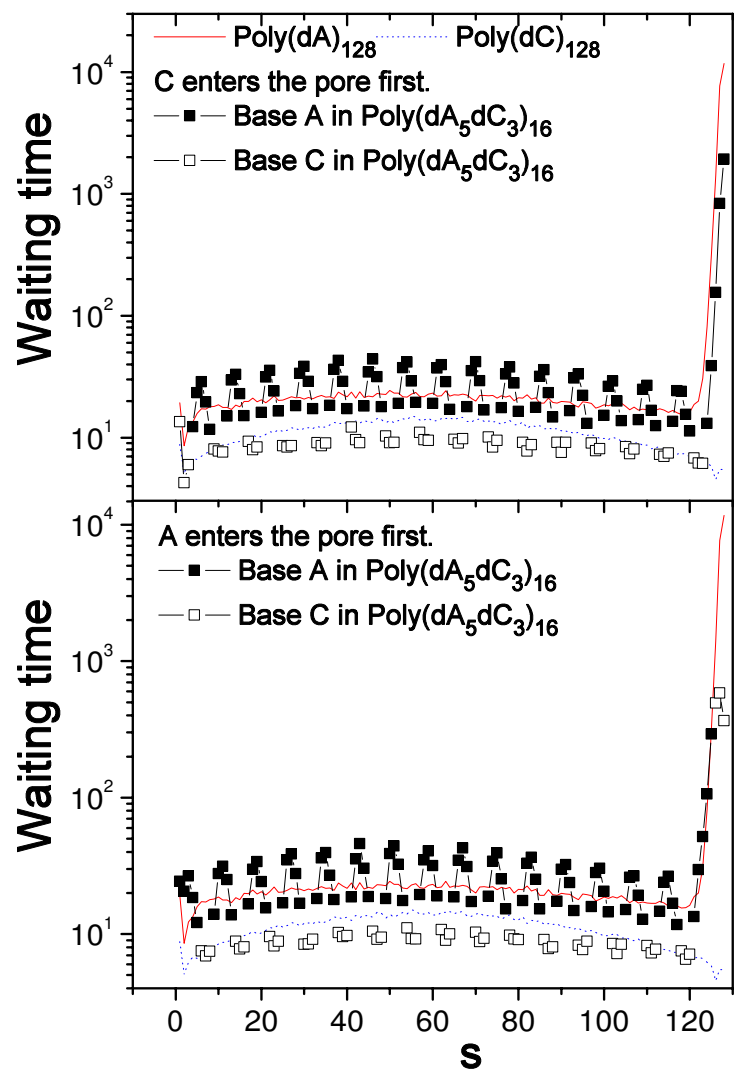

FIG. 6. (Color online) The waiting time distribution for poly $\left(\mathrm{dA}_{5} \mathrm{dC}_{3}\right)_{16}$. Here, $\varepsilon_{p A}=3.0, \varepsilon_{p C}=1.0$, and the chain length $N$ $=128$. 


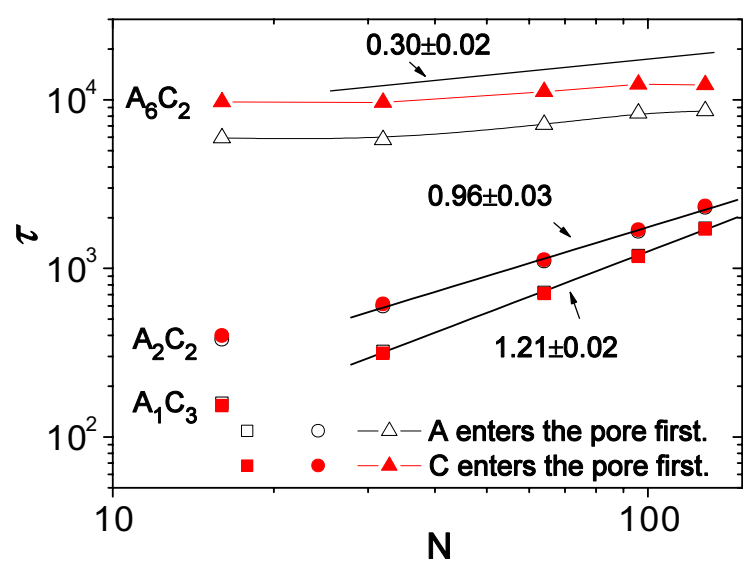

FIG. 7. (Color online) Translocation time as a function of the chain length for different sequences. Here, $\varepsilon_{p A}=3.0$, and $\varepsilon_{p C}=1.0$.

a maximum because of the increase in the activation energy. Thus the number of peaks is exactly equal to $N /(m+n)$, which is the periodicity of the sequences (cf. Fig. 6).

\section{Translocation time as a function of the chain length for different sequences}

Previously, we observed that the translocation time of homopolymers with pure repulsive interaction with the pore scales as $\tau \propto N^{2 \nu}$ with the chain length for relatively short chains $(N<200)[54,55]$, where $\nu=0.75$ is the Flory exponent in 2D [77,78]. For heteropolymers consisting of two types of monomers that have a pure repulsive interaction with the pore and distinguished only by the magnitude of the driving force that they experience inside the pore, we find that these scaling properties remain valid with arbitrary repeat unit [57]. This could be easily understood by noting that at a higher level of coarse-graining, the microstructure of the chain is irrelevant as far as universal scaling properties are concerned.

When attractive interaction is introduced between the monomers and the wall of the pore, we have found recently that while the scaling $\tau \propto N^{2 v}$ is still valid for poly $(\mathrm{dC})$ with the weaker attraction, the translocation time $\tau$ shows nonmonotonic behavior as a function of the chain length for poly $(\mathrm{dA})$ due to the strong attractive force between poly $(\mathrm{dA})$ and the pore [58]. This leads to an interesting question on the translocation time $\tau$ scales with $N$ for different sequences $A_{m} C_{n}$ in heteropolymers considered in this work. In Fig. 7 we plot the results showing how the translocation time scales as a function of chain length $N$ for different repeat structures $A_{1} C_{3}, A_{2} C_{2}, A_{3} C_{1}$, and $A_{6} C_{2}$. For $A_{3} C_{1}$ and $A_{6} C_{2}$, the translocation time $\tau$ depends on the orientation, but the orientation does not affect the qualitative dependence of $\tau$ on $N$.

For $A_{1} C_{3}$, the scaling exponent is 1.21 which is close to $2 \nu$. However, with increasing block length of the base $A$, the effective scaling exponent decreases. For $A_{2} C_{2}$, it is 0.96 and it is less than 0.30 for $A_{3} C_{1}, A_{6} C_{2}$. The dependence on the length of DNA is due to the change from the nonactivated regime for weak attractive or pure repulsive interaction to an activated regime for strong attractive interaction. It would be desirable to have measurement made over a larger range of composition to critically test our predictions for the effective scaling exponent.

\section{CONCLUSIONS}

In this work, we have investigated the dynamics of DNA translocation through a nanopore driven by an external force using 2D Langevin dynamics simulations with an aim to understand how various aspects of the translocation dynamics is affected by a specific sequence. We have considered coarse-grained models of hetero-DNAs consisting of two types of bases $A$ and $C$, which are differentiated by the different base-pore interactions, such as a strong attractive force between the pore and the base $A$ and a weaker attractive force between the pore and the base $C$. From a series of studies on polymers with sequences $A_{m} C_{n}$, we find the exponential dependence of the translocation time on $f_{C}$, the volume fraction of the base $C$. For longer sequences with $f_{C}$ $\leqslant 0.5$ the translocation time strongly depends on the orientation of DNA, namely the condition which base enters the pore first. These results are interpreted according to the waiting times of the individual bases. We find that the waiting time displays intriguing fringe patterns that can be understood from the process of emptying the more attractive subblocks from the pore. This also leads to a very simple qualitative understanding of the orientational dependence of the translocation time depending on which base enters the pore first. Furthermore, based on the waiting time distribution, the sequence lengths $m, n$ and their periodicity can be distinguished.

One may wonder to what extent our studies are relevant to bringing this observation into practice and to develop a "fast sequencing machine"? Among the most important conclusions here is that the waiting time distribution of individual monomers can be used to identify sequences in heteropolymers. It is worth noting in this context that the ionization potentials for different polynucleotides are different. Therefore, it is plausible that laser-induced attractive interactions along with fluorescence spectroscopy could be used to make a device that will detect either the waiting time or the number of monomers inside the pore as a function of time.

\section{ACKNOWLEDGMENTS}

This work has been supported in part by The Academy of Finland through its Center of Excellence COMP and TransPoly Consortium grants. We wish to thank Center for Scientific Computing-CSC Ltd. for allocation of computer time. 
[1] J. J. Kasianowicz, E. Brandin, D. Branton, and D. W. Deamer, Proc. Natl. Acad. Sci. U.S.A. 93, 13770 (1996).

[2] A. Meller, J. Phys.: Condens. Matter 15, R581 (2003).

[3] M. Akeson, D. Branton, J. J. Kasianowicz, E. Brandin, and D. W. Deamer, Biophys. J. 77, 3227 (1999).

[4] A. Meller, L. Nivon, E. Brandin, J. A. Golovchenko, and D. Branton, Proc. Natl. Acad. Sci. U.S.A. 97, 1079 (2000).

[5] A. Meller, L. Nivon, and D. Branton, Phys. Rev. Lett. 86, 3435 (2001).

[6] A. Meller and D. Branton, Electrophoresis 23, 2583 (2002).

[7] M. Wanunu and A. Meller, Nano Lett. 7, 1580 (2007).

[8] S. M. Iqbal, D. Akin, and R. Bashir, Nat. Nanotechnol. 2, 243 (2007).

[9] A. F. Sauer-Budge, J. A. Nyamwanda, D. K. Lubensky, and D. Branton, Phys. Rev. Lett. 90, 238101 (2003).

[10] J. Mathe, H. Visram, V. Viasnoff, Y. Rabin, and A. Meller, Biophys. J. 87, 3205 (2004).

[11] S. E. Henrickson, M. Misakian, B. Robertson, and J. J. Kasianowicz, Phys. Rev. Lett. 85, 3057 (2000).

[12] J. L. Li, D. Stein, C. McMullan, D. Branton, M. J. Aziz, and J. A. Golovchenko, Nature (London) 412, 166 (2001).

[13] J. L. Li, M. Gershow, D. Stein, E. Brandin, and J. A. Golovchenko, Nature Mater. 2, 611 (2003).

[14] D. Fologea, J. Uplinger, B. Thomas, D. S. McNabb, and J. L. Li, Nano Lett. 5, 1734 (2005).

[15] O. V. Krasilnikov, C. G. Rodrigues, and S. M. Bezrukov, Phys. Rev. Lett. 97, 018301 (2006).

[16] U. F. Keyser, J. B. M. Koelman, S. van Dorp, D. Krapf, R. M. M. Smeets, S. G. Lemay, N. H. Dekker, and C. Dekker, Nat. Phys. 2, 473 (2006).

[17] U. F. Keyser, J. van der Does, C. Dekker, and N. H. Dekker, Rev. Sci. Instrum. 77, 105105 (2006).

[18] C. Dekker, Nat. Nanotechnol. 2, 209 (2007).

[19] E. H. Trepagnier, A. Radenovic, D. Sivak, P. Geissler, and J. Liphardt, Nano Lett. 7, 2824 (2007).

[20] A. J. Storm, J. H. Chen, X. S. Ling, H. W. Zandbergen, and C. Dekker, Nature Mater. 2, 537 (2003).

[21] A. J. Storm, C. Storm, J. H. Chen, H. W. Zandbergen, J. -F. Joanny, and C. Dekker, Nano Lett. 5, 1193 (2005).

[22] A. J. Storm, J. H. Chen, H. W. Zandbergen, and C. Dekker, Phys. Rev. E 71, 051903 (2005).

[23] S. M. Simon, C. S. Peskin, and G. F. Oster, Proc. Natl. Acad. Sci. U.S.A. 89, 3770 (1992).

[24] W. Sung and P. J. Park, Phys. Rev. Lett. 77, 783 (1996).

[25] P. J. Park and W. Sung, J. Chem. Phys. 108, 3013 (1998).

[26] E. A. diMarzio and A. L. Mandell, J. Chem. Phys. 107, 5510 (1997).

[27] M. Muthukumar, J. Chem. Phys. 111, 10371 (1999).

[28] M. Muthukumar, J. Chem. Phys. 118, 5174 (2003).

[29] C. Y. Kong and M. Muthukumar, J. Chem. Phys. 120, 3460 (2004).

[30] M. Muthukumar, Annu. Rev. Biophys. Biomol. Struct. 36, 435 (2007).

[31] S. Ghosal, Phys. Rev. Lett. 98, 238104 (2007).

[32] S. Ghosal, Phys. Rev. E 74, 041901 (2006).

[33] M. G. Gauthier and G. W. Slater, Eur. Phys. J. E 25, 17 (2008); S. Guillouzic and G. W. Slater, Phys. Lett. A 359, 261 (2006).

[34] A. Lzmitli, D. C. Schwartz, M. D. Graham, and J. J. de Pablo, J. Chem. Phys. 128, 085102 (2008).
[35] M. Fyta, S. Melchionna, S. Succi, and E. Kaxiras, Phys. Rev. E 78, 036704 (2008).

[36] J. B. Heng, A. Aksimentiev, C. Ho, P. Marks, Y. V. Grinkova, S. Sligar, K. Schulten, and G. Timp, Nano Lett. 5, 1883 (2005).

[37] J. B. Heng, A. Aksimentiev, C. Ho, P. Marks, Y. V. Grinkova, S. Sligar, K. Schulten, and G. Timp, Biophys. J. 90, 1098 (2006).

[38] U. Bockelmann, and V. Viasnoff, Biophys. J. 94, 2716 (2008).

[39] D. K. Lubensky and D. R. Nelson, Biophys. J. 77, 1824 (1999).

[40] Y. Kafri, D. K. Lubensky, and D. R. Nelson, Biophys. J. 86, 3373 (2004).

[41] E. Slonkina and A. B. Kolomeisky, J. Chem. Phys. 118, 7112 (2003).

[42] T. Ambjornsson, S. P. Apell, Z. Konkoli, E. A. DiMarzio, and J. J. Kasianowicz, J. Chem. Phys. 117, 4063 (2002).

[43] R. Metzler and J. Klafter, Biophys. J. 85, 2776 (2003).

[44] T. Ambjornsson and R. Metzler, Phys. Biol. 1, 77 (2004).

[45] T. Ambjornsson, M. A. Lomholt, and R. Metzler, J. Phys.: Condens. Matter 17, S3945 (2005).

[46] A. Baumgartner and J. Skolnick, Phys. Rev. Lett. 74, 2142 (1995).

[47] J. Chuang, Y. Kantor, and M. Kardar, Phys. Rev. E 65, 011802 (2001).

[48] Y. Kantor and M. Kardar, Phys. Rev. E 69, 021806 (2004).

[49] Y. Kantor and M. Kardar, Phys. Rev. E 76, 061121 (2007); C. Chatelain, Y. Kantor, and M. Kardar, ibid. 78, 021129 (2008).

[50] J. K. Wolterink, G. T. Barkema, and D. Panja, Phys. Rev. Lett. 96, 208301 (2006); D. Panja, G. T. Barkema, and R. C. Ball, J. Phys.: Condens. Matter 19, 432202 (2007); 20, 075101 (2008); Biophys. J. 94, 1630 (2008); H. Vocks, D. Panja, G. T. Barkema, and R. C. Ball, J. Phys.: Condens. Matter 20, 095224. (2008)

[51] J. L. A. Dubbeldam, A. Milchev, V. G. Rostiashvili, and T. A Vilgis, Phys. Rev. E 76, 010801(R) (2007); , Europhys. Lett. 79, 18002 (2007).

[52] A. Milchev, K. Binder, and A. Bhattacharya, J. Chem. Phys. 121, 6042 (2004).

[53] K. F. Luo, T. Ala-Nissila, and S. C. Ying, J. Chem. Phys. 124 034714 (2006).

[54] K. F. Luo, I. Huopaniemi, T. Ala-Nissila, and S. C. Ying, J. Chem. Phys. 124, 114704 (2006).

[55] I. Huopaniemi, K. F. Luo, T. Ala-Nissila, and S. C. Ying, J. Chem. Phys. 125, 124901 (2006).

[56] I. Huopaniemi, K. F. Luo, T. Ala-Nissila, and S. C. Ying, Phys. Rev. E 75, 061912 (2007).

[57] K. F. Luo, T. Ala-Nissila, S. C. Ying, and A. Bhattacharya, J. Chem. Phys. 126, 145101 (2007).

[58] K. F. Luo, T. Ala-Nissila, S. C. Ying, and A. Bhattacharya, Phys. Rev. Lett. 99, 148102 (2007)

[59] K. F. Luo, T. Ala-Nissila, S. C. Ying, and A. Bhattacharya, Phys. Rev. Lett. 100, 058101 (2008).

[60] K. F. Luo, S. T. T. Ollila, I. Huopaniemi, T. Ala-Nissila, P. Pomorski, M. Karttunen, S. C. Ying, and A. Bhattacharya, Phys. Rev. E 78, 050901(R) (2008); K. F. Luo, T. Ala-Nissila, S. C. Ying, P. Pomorski, and M. Karttunen, e-print arXiv:0709.4615..

[61] S.-S. Chern, A. E. Cardenas, and R. D. Coalson, J. Chem. Phys. 115, 7772 (2001) 
[62] H. C. Loebl, R. Randel, S. P. Goodwin, and C. C. Matthai, Phys. Rev. E 67, 041913 (2003).

[63] R. Randel, H. C. Loebl, and C. C. Matthai, Macromol. Theory Simul. 13, 387 (2004).

[64] Y. Lansac, P. K. Maiti, and M. A. Glaser, Polymer 45, 3099 (2004).

[65] C. Y. Kong and M. Muthukumar, Electrophoresis 23, 2697 (2002).

[66] Z. Farkas, I. Derenyi, and T. Vicsek, J. Phys.: Condens. Matter 15, S1767 (2003).

[67] P. Tian and G. D. Smith, J. Chem. Phys. 119, 11475 (2003). We note that the results here contradict both experiments as well as other simulation studies.

[68] D. Wei, W. Yang, X. Jin, and Q. Liao, J. Chem. Phys. 126, 204901 (2007); Y. D. He, H. J. Qian, Z. Y. Lu, and Z. S. Li, Polymer 48, 3601 (2007); Y. C. Chen, C. Wang, and M. Luo, J. Chem. Phys. 127, 044904 (2007); Y. J. Xie, H. Y. Yang, H. T. Yu, Q. W. Shi, X. P. Wang, and J. Chen, ibid. 124, 174906 (2006).

[69] R. Zandi, D. Reguera, J. Rudnick, and W. M. Gelbart, Proc. Natl. Acad. Sci. U.S.A. 100, 8649 (2003).
[70] S. Kotsev and A. B. Kolomeisky, J. Chem. Phys. 125, 084906 (2006).

[71] S. Tsuchiya and A. Matsuyama, Phys. Rev. E 76, 011801 (2007).

[72] B. Alberts et al., Molecular Biology of the Cell (Garland, New York, 1994).

[73] M. P. Allen and D. J. Tildesley, Computer Simulation of Liquids (Oxford University Press, Oxford, 1987).

[74] S. B. Smith, Y. Cui, and C. Bustamante, Science 271, 795 (1996); M. C. Murphy, I. Rasnik, W. Cheng, T. M. Lohman, and T. Ha, Biophys. J. 86, 2530 (2004).

[75] $W$ is measured from the center of the wall particles. For a value of $W=3 \sigma$, the actual separation between the outermost boundaries of the two walls is $2 \sigma$.

[76] D. L. Ermak and H. Buckholz, J. Comput. Phys. 35, 169 (1980).

[77] P. G. de Gennes, Scaling Concepts in Polymer Physics (Cornell University Press, Ithaca, NY, 1979).

[78] M. Doi and S. F. Edwards, The Theory of Polymer Dynamics (Clarendon, Oxford, 1986). 